\title{
Immunostimulatory DNA sequences function as T helper-1-promoting adjuvants
}

\author{
Mark Roman ${ }^{1}$, Elena Martin-Orozco ${ }^{1}$, Justin S. Goodman ${ }^{1}$, Minh-Duc NGUyen ${ }^{1}$, \\ Yukio Sato ${ }^{2}$, Arash Ronaghy ${ }^{1}$, Richard S. Kornbluth ${ }^{3}$, Douglas D. Richman ${ }^{4}$, \\ DENNIS A. CARSON ${ }^{1} \&$ EYAL RAZ \\ 'Dynavax Technologies Corporation, 3099 Science Park Road, Suite 200, San Diego, California 92121 \\ ${ }^{2}$ Department of Internal Medicine II, Fukushima Medical College, Fukushima 960-12, Japan \\ ${ }^{3}$ Department of Medicine, University of California San Diego, 9500 Gilman Drive, La Jolla, Califormia 92093-0679 \\ 'San Diego Veterans Affairs Medical Center and Departments of Medicine and Pathology, University of California \\ San Diego, 9500 Gilman Drive, La Jolla, California 92093-0679 \\ Correspondence should be addressed to E.R.
}

\begin{abstract}
An adjuvant role for certain short bacterial immunostimulatory DNA sequences (ISSs) has recently been proposed on the basis of their ability to stimulate T helper-1 (Th1) responses in gene-vaccinated animals. We report here that noncoding, ISS-enriched plasmid DNAs or ISS oligonucleotides (ISS-ODNs) potently stimulate immune responses to coadministered antigens. The ISS-DNAs suppress IgE synthesis, but promote IgG and interferon- $\gamma($ IFN $-\gamma$ ) production. They furthermore initiate the production of IFN- $\gamma$, IFN- $\alpha$, IFN- $\beta$, and interleukins 12 and 18, all of which foster Th1 responses and enhance cell-mediated immunity. Consideration should be given to adding noncoding DNA adjuvants to inactivated or subunit viral vaccines that, by themselves, provide only partial protection from infection.
\end{abstract}

Subunit or inactivated virus vaccines typically induce T helper-2 (Th2) immune responses, with high titers of neutralizing antibodies but without significant cell-mediated immunity. In contrast, intradermal and intramuscular vaccination with naked pDNA stimulates immune responses with a Th1 bias ${ }^{1-4}$, with the expansion of $\mathrm{CD} 4^{+} \mathrm{T}$ cells producing IFN- $\gamma$ and cytotoxic $\mathrm{CD}^{+} \mathrm{T}$ cells. The Th1 response to gene vaccination is maximal when the pDNA backbone contains short (6 base pairs) immunostimulatory DNA sequences (ISSs) with CpG motifs ${ }^{5}$. These sequences are 20 times as common in bacterial as in mammalian DNA (ref. 6). Simultaneous injection of ISS-deficient pDNA encoding a nominal antigen with a noncoding, ISS-enriched pDNA also fosters a Th1 response, indicating that the ISS-enriched pDNA exerts an adjuvant effect ${ }^{5}$. However, gene vaccines generally stimulate antibody production inefficiently when compared with matching protein vaccines. This deficiency probably results from the small number of transfected and/or antigen-producing cells, and from the inhibition of antigen synthesis by IFN- $\alpha$ and IFN- $\beta$ induced by the ISSs in the pDNA backbone $e^{5}$.

Given that the ISSs in pDNA have an immunostimulatory (adjuvant) effect in gene-vaccinated animals, we hypothesized that ISS-enriched pDNA or ISS-oligodeoxynucleotide (ISS-ODN) might also deliver the necessary stimulatory signals for Th1 induction to a coinjected protein antigen. In principle, protein/ISS-pDNA or protein/ISS-ODN coadministration could combine the advantages of the Th1 response achieved by naked DNA immunization with the high levels of antibody generated by protein vaccines. Experiments were therefore designed to determine whether the coadministration strategy could successfully improve upon the outcome of either gene or protein vaccination, as well as to establish the underlying immunostimulatory mechanisms of the DNA adjuvant'.
Immunostimulatory DNA sequences enhance anti- $\beta$-gal antibody response

Mice were injected intradermally once with $\beta$-galactosidase ( $\beta$ gal) protein, together with either genomic Escherichia coli or calf thymus DNA. Antibody isotype responses to the injected antigen were then assessed by enzyme-based immunosorbent assay (ELISA). Coadministered bacterial ( $E$. coli) DNA enhanced IgG2a anti- $\beta$-gal antibody production, suggestive of a Th1 response, whereas calf thymus DNA had no effect (Fig. 1a). To identify DNA sequences that induced or enhanced the IgG2a response, mice were immunized with various pDNAs as adjuvants. The ampicillin resistance gene $(a m p R)$ contains two repeats of the 5'-AACGTT-3' ISS; the kanamycin resistance gene $(k a n R)$ contains none (Fig. 2). The coadministration of the noncoding kanR-based vector, $\mathrm{pKCB}$, with $\beta$-gal induced no higher levels of $\operatorname{IgG} 2 \alpha$ than $\beta$-gal alone; however, coinjecting the noncoding ampR-based vectors, $\mathrm{pACB}$ or $\mathrm{pUC19}$, with $\beta$-gal generated high levels of IgG2a (Fig. $1 b$ ).

To determine the role of the 5'-AACGTT-3' ISS on anti- $\beta$-gal IgG2a production, the CMV promoter region (which contains other potential ISSs, Fig. 2), through the poly(A) ${ }^{+}$tail sequence of the parental $\mathrm{pKCB}$ vector was deleted, and the resultant vector underwent various modifications. A new set of vectors, the pKISS-0 through pKISS-3, was then created, which have zero to three repeats of the 5'-AACGTT-3' ISS, respectively (K for kanR gene and ISS for immunostimulatory DNA sequences). The coinjection of pKISS- 0 with $\beta$-gal did not promote IgG2a production, whereas the coinjection of pKISS-1 through pKISS- 3 with the antigen induced anti- $\beta$-gal IgG 2 a levels equivalent to those obtained with the codelivery of the ampR-based vectors $\mathrm{pACB}$ or pUC19 (Fig. 1c). The coinjection of $\beta$-gal with single-stranded ISS phosphorothioate ODN strongly enhanced the basal anti- $\beta$-gal 

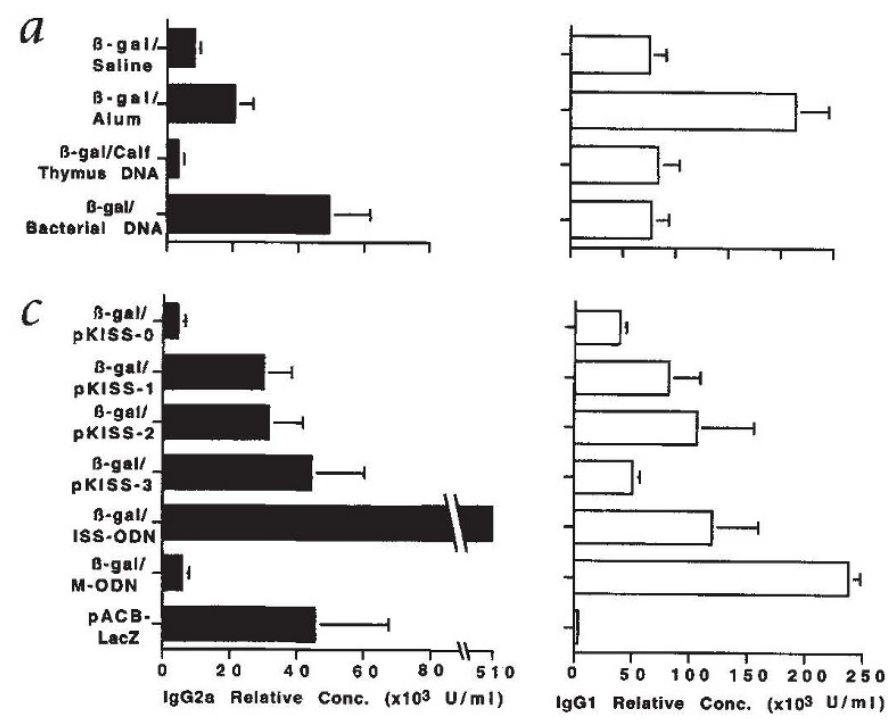

$b$
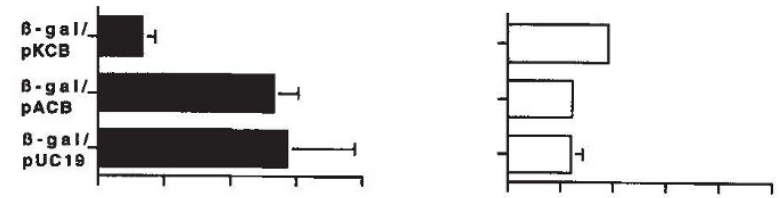

Fig. 1 Isotype responses to $\beta$-gal in BALB/C mice immunized once with various $\beta-g a l / D N A(s)$ combinations. The sixth week post-immunization isotype responses following a single i.d. injection are shown for $a, b$ and $c$. $a$, The effect on anti- $\beta$-gal lgG2a response by bacterial $(E$. coli) versus calf thymus DNA codelivered with $\beta$-gal. $b$, The effect of coinjection of $\beta$-gal with ampR-related pDNAs (pACB and pUC19) versus kanR pDNA (pKCB) on $\lg \mathrm{G} 2 \mathrm{a}$ and $\lg \mathrm{G} 1$ anti- $\beta$-gal antibody responses. $C$, The outcome of the coinjection of the modified kanR-based pDNAs, pKISS-0 through pKISS-3, as well as the phosphorothioate ISS-ODN and M-ODN on the subsequent $\operatorname{lgG} 2 \mathrm{a}$ and $\lg \mathrm{G} 1$ anti- $\beta$-gal antibody production. $d$, Isotype responses to $\beta$ gal after three immunizations ( 2 weeks apart). The isotype levels obtained with the $\beta$-gal/pDNA combinations were compared with those of the matching gene vaccine, PACB-LacZ (the sixth week antibody responses are shown). $\beta$-gal/ISS-pDNA coadministration generated higher anti- $\beta$-gal IgC levels than the protein or the matching gene vaccine. ISS-pDNA also similarly amplified the anti-ovalbumin IgC response in ovalbumin/ISS-pDNA coimmunized mice (data not shown).

As controls, injection of pUC19, pACB or pKCB alone i.d. did not induce any anti- $\beta$-gal activity. Intradermal administration of $\beta$-gal and pUC19 into two different sites (at the base of the tail and at the nape, respectively) or at the base of the tail, 1 week apart, did not lead to anti- $\beta$-gal IgG2a induction (data not shown). Results are the means \pm s.e.m. for four mice per group.
IgG2a levels induced by $\beta$-gal alone; however, the coinjected mutated (M) ODN (in which each CG of the ISS-ODN was replaced with $\mathrm{GG}$ ) did not. In contrast the coinjection of $\beta$-gal with ISS phosphodiester ODN did not alter the basal anti- $\beta$-gal IgG2a levels, probably because of the short half-life of the phosphodiester ODNs in vivo (data not shown). Immunization of animals three times with $\beta$-gal/ISS-pDNAs amplified their anti- $\beta$-gal IgG2a antibodies (9-fold for pKISS-3 and 12.5-fold for pUC19) in comparison with $\beta$-gal alone or with pKISS-0, the ISS-deficient pDNA (Fig. $1 d$ ). The coadministration of $\beta$-gal with the various ISS-pDNAs elicited higher anti- $\beta$-gal IgG antibodies than $\beta$-gal alone or the matching gene vaccine, PACB-LacZ (Fig. 1d).

\section{ISS-DNAs enhance anti-hemagglutinin antibody response}

The addition of pKISS-3 or ISS phosphorothioate ODN to a commercial, inactivated subunit human influenza vaccine upregulated by three and by eightfold, respectively, the IgG antibody responses to the viral hemagglutinins (HGNs) (Fig. 3). These results established that the adjuvant effect of ISS-DNAs (ODN or pDNA) also applies to common vaccines in clinical use.

\section{Cytokine profile of b-gal/Iss-DNAs injected mice}

T helper-1-type cells characteristically produce IFN- $\gamma$ after antigen restimulation ${ }^{8}$. Protein/ISS-pDNA coimmunization induced this pattern of cytokine release (Table 1). Antigen-stimulated $\mathrm{CD}^{+}$ splenocytes from mice immunized with $\beta$-gal/pKISS-3 and $\beta$ gal/pUC19 generated high levels of IFN- $\gamma$, whereas $\mathrm{CD}^{+}$cells from mice immunized with $\beta$-gal or $\beta$-gal/pKISS- 0 synthesized only trace amounts. Compared with $\mathrm{CD}^{+}$cells from gene-vaccinated mice, the $\mathrm{T}$ cells from protein/ISS-pDNA covaccinated ani- mals produced higher levels of IL-4 (Table 1). However, the ratio of IFN- $\gamma$ to IL- 4 was consistent with a shift of the anti- $\beta$-gal response toward a Th1 phenotype.

\section{ISS-DNAs suppress IgE antibody formation}

Immunoglobulin E production is a consequence of extreme Th2 responses ${ }^{8}$ and can lead to potentially fatal anaphylactic reactions to protein vaccines. To evaluate whether Th1 stimulation by antigen/ISS-DNAs (ODN or pDNA) coimmunization could inhibit the subsequent anti- $\beta$-gal IgE synthesis, mice were first primed with $\beta$-gal/pKISS-0, $\beta$-gal/pKISS-3, $\beta$-gal/M-ODN (phosphorothioate), $\beta$-gal/ISS-ODN (phosphorothioate), or saline. Mice were then boosted intraperitoneally 4 weeks later with $\beta$-gal in alum. The mice primed with $\beta$-gal/ISS-DNAs were "resistant" to IgE inductions as they did not generate any significant anti- $\beta$ -

Table 1 Cytokine profile of in vitro $\beta$-gal-stimulated CD4 ${ }^{+}$ splenocytes from mice immunized with $\beta$-gal, $\beta$-gal/pDNA combinations or with the matching gene vaccine, $\mathrm{PACB}-\mathrm{LaCZ}$

\begin{tabular}{lcc} 
Immunization & $\mathrm{mlL}-4(\mathrm{pg} / \mathrm{ml})$ & $\mathrm{mIFN}-\gamma(\mathrm{ng} / \mathrm{ml})$ \\
Naive & $<10$ & $<4$ \\
pKISS-3 & $<15$ & $<10$ \\
$\beta$-gal & $158 \pm 60$ & $9 \pm 4$ \\
$\beta$-gal/pKISS-O & $144 \pm 57$ & $12 \pm 6$ \\
$\beta$-gal/pKISS-3 & $136 \pm 46$ & $54 \pm 16$ \\
$\beta$-gal/pUC19 & $167 \pm 51$ & $95 \pm 11$ \\
pACB-LacZ & $16 \pm 3$ & $270 \pm 108$ \\
\hline
\end{tabular}

Mice were killed 2 weeks after the last immunization. Only background levels of IFN- $y$ or $\mathrm{LL}-4$ were detected in the supernatants of $\mathrm{CD}^{+}$splenocytes unstimulated in vitro with $\beta$-gal. Results are the means \pm s.e.m. of four mice per group. 


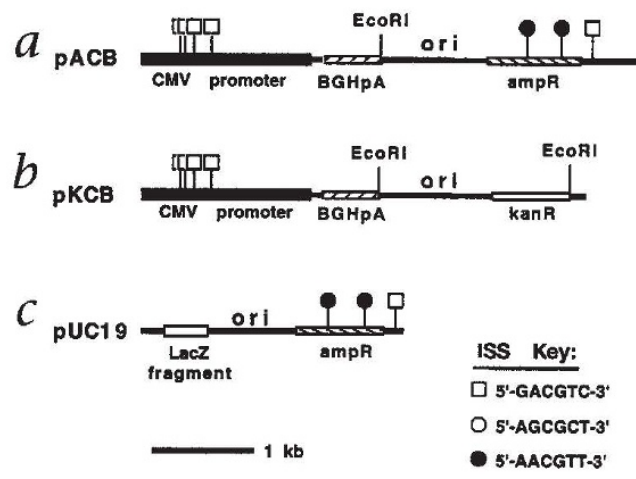

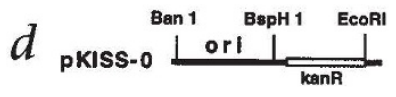
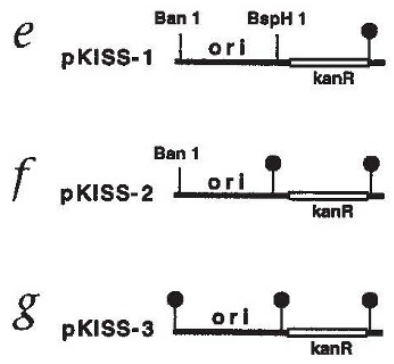

Fig. 2 Localization of the ISSs on the various pDNA(s) used in this study. The PACB is a pUC19-based pDNA and was previously described in detail'. The $\mathrm{pKCB}$ vector was constructed by replacing the 1542-bp ampR (BspHI-HindIII) with a 1042-bp kanR-containing fragment (BspHI-HindIII). The ISS-ODN flanked by EcoRI-compatible overhangs (sense, 5'-AATTGAACGTTCGC-3', antisense, 5'-AATTCCGAACGTTC-3') was ligated into a unique EcoRI site of PACB, 3 ' to the $B G H p A$ sequence. This resulted in the disruption of the EcoRI site, and the creation of a new Psp1406I restriction site (AACGTT). The ISS-containing region was then subcloned $(B s p H I-B a m H I)$ into the pKISS-O to create pKISS-1. The vectors pKISS-2 and pKISS-3 were constructed by ligation of the same ISS-ODN into pKISS-1 and pKISS-2, respectively, at a different ECoRI site. gal IgE antibodies in response to a subsequent boosting with $\beta$ gal/alum, as opposed to mice primed with $\beta$-gal/pKISS- 0 or $\beta$ gal/M-ODN, or saline-injected animals (Fig. 4). This inhibition of specific IgE induction is similar to that observed upon gene immunization ${ }^{3}$.

\section{ISS-DNAs activate macrophages to produce Th1-promoting cytokines}

Specialized antigen-presenting cells (APCs) are susceptible to in vivo transfection with pDNA (ref. 9, 10) and dictate the specificity of immune responses after naked DNA immunization". Transfection of fresh human macrophages with ISS-pDNA or with ISS-ODN, but not with ISS-deficient pDNA or M-ODN, increased IFN- $\alpha$, IFN- $\beta$, IL-12, and IL-18 mRNA levels (Fig. 5, $a-d$ ). These cytokines of the innate response are established inducers of IFN- $\gamma$ synthesis ${ }^{12-15}$. Incubation of human macrophages with IFN- $\alpha$ raised IL-12 and IL-18 mRNA levels, suggesting an amplifying role for IFN- $\alpha$ in ISS-induced stimulation (Fig. 5, $e$ and $f$ ) and possibly in the induction of a Th1 response in this system. Furthermore, transfection of human peripheral blood mononuclear cells with ISS-ODN, but not with M-ODN, resulted in the production of IFN- $\gamma$ (Table 2 ) as well as IFN- $\alpha$, IFN- $\beta$ and IL- 12 by the transfected cells (Table 2 and Fig. 5). It is interesting that transfection of enriched peripheral human $\mathrm{CD}^{+}$or $\mathrm{CD} 8^{+}$, as well as the human $\mathrm{T}$ cell lines CEM and Jurkat, under the same conditions, did not result in detectable secretion of IFN- $\gamma$. Taken together, these data suggest a key role for macrophages in the secretion of the IFN- $\gamma$ inducers, and NK cells for the secretion of IFN- $\gamma$ (ref. 16, 17).

\section{Discussion}

Compared with conventional protein vaccines, gene vaccines usually induce lower levels of antibodies, but stronger cellular immunity. Insofar as neutralizing antibodies are the first line of defense against viruses, bacteria and their toxins, gene immunization alone may not be an optimal approach for prevention of infectious diseases. However, cellular immune responses are a critical component of the host's response to infection and may be required for recovery. Unfortunately, neither inactivated virus nor subunit vaccines induce both vigorous cellular immunity and neutralizing antibodies. This limitation may have hampered the development of recombinant vaccines, as exemplified by the prominent failure of vaccines for HIV (ref. 18) and herpes simplex virus 2 (ref. 19). The present experiments show that coadministration of a standard protein vaccine with ISS-enriched DNA elicits antibody titers several times those of the matching gene vaccine. This combination additionally activates cell-mediated immunity, as assessed by production of IFNs $\alpha, \beta$, and $\gamma$. The simple addition of the DNA adjuvant to a commercial, inactivated subunit influenza vaccine greatly increased its immunogenicity. Thus, the coadministration of the DNA adjuvant with influenza virus vaccine or with other viral antigens should enhance protection and could reduce morbidity and mortality of vaccinated hosts from invading pathogen(s). Moreover, in atopic diseases, the allergen/ISS-DNA coimmunization may elicit higher titers of blocking IgG antibodies, as well as Th1 immunity. Both effects should contribute to the suppression of immediate hypersensitivity ${ }^{3}$ and the late-phase reactions upon reexposure to the allergen, by suppressing IgE synthesis and eosinophil recruitment, respectively ${ }^{4}$.

Although intradermal and intramuscular gene immunization stimulates a Th1 immune response $\mathrm{e}^{1-3}$, gene immunization of mice by biolistic delivery into the skin with pDNA-coated gold microspheres induces immune responses with a Th2 bias ${ }^{20,21}$. Compared with intradermal or intramuscular gene immunization with naked pDNA, vaccination with gold microspheres uses $1 / 100$ th as much pDNA (ref. 22), and it might follow systemic dispersal of the delivered pDNA and the transfected cells. In these circumstances, the low dose of the delivered pDNA probably is insufficient to provide the local adjuvant effect that may be necessary to trigger Th1 immunity. On the other hand, the local production of IFNs $\alpha, \beta$, and $\gamma$ as well as IL-12 and IL-18 within the skin or muscle by higher doses of ISS-DNA may induce a specific cytokine milieu which promotes Th1 and cell-mediated immunity to the encoded antigen.
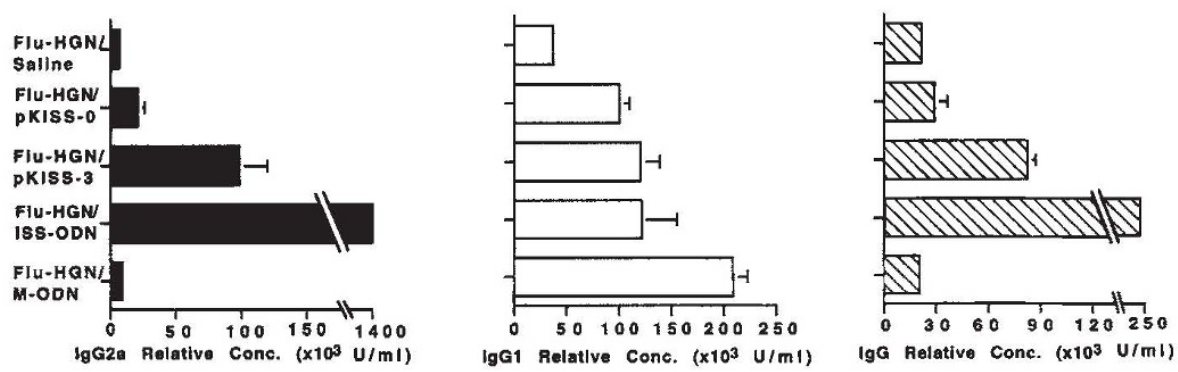

Fig. 3 The antibody response to influenza virus hemagglutinins (HGN) administration, alone or with various ISS-DNAs or ISS-deficient DNAs. ISS-ODN and pKISS-3 modified and upregulated the anti$\mathrm{HGN} \lg \mathrm{G} 2 \mathrm{a}$ and $\lg G$ responses. Results are the means \pm s.e.m. for four mice per group. 


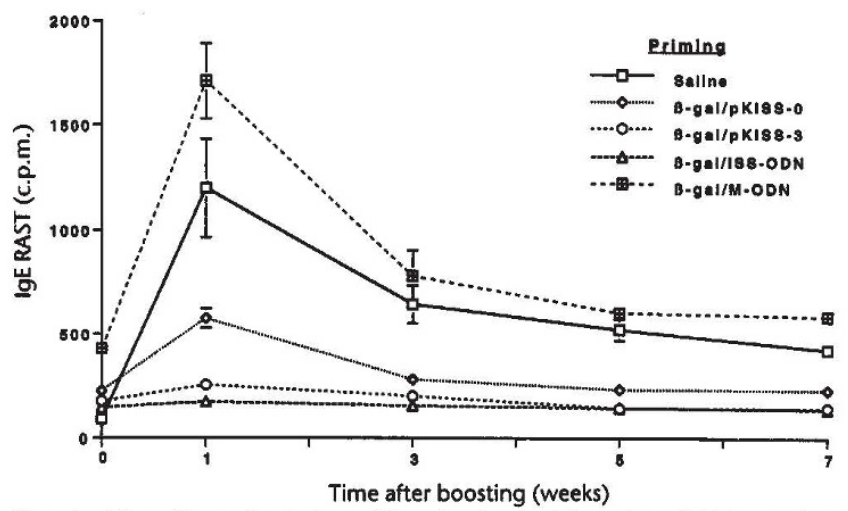

Fig. 4 The effect of priming of $\beta$-gal mixed with various DNAs on the subsequent anti- $\beta$-gal IgE response after i.p. boosting with $\beta$-gal in alum. Mice primed with $\beta$-gal/pKISS-3 and $\beta$-gal/ISS-ODN did not generate significant anti- $\beta$-gal IgE antibodies as opposed to the other groups. Results are the means \pm s.e.m. for four mice per group. $P<0.05$ for $\beta$-gal/pKISS- 3 or the $\beta$ gal/ISS-ODN versus saline, $\beta$-gal, $\beta$-gal/M-ODN or versus $\beta$-gal/pKISS-3. The mice in these experiments displayed a strong anti- $\beta$-gal IgC response before i.p. boosting and higher titers post boosting (data not shown). The differences in the IgE levels between the $\beta$-gal/pKISS- 0 and the $\beta$-gal/MODN may be attributed to some nonpalindromic $\mathrm{CpG}$ motifs ${ }^{27}$ in the pKISS0 vector.

The mechanism by which gene vaccination induces a Th1 response $^{1-3}$ is still unclear. The data presented here support the assumption that this response is the consequence of the activation of the innate immune response by the ISSs in the pDNA backbone $^{5}$, rather than by the low dose of antigen intracellularly produced. The activation products of the ISSs, that is, type-I IFNs, together with IL-12 and IL-18, are established inducers of IFN- $\gamma$ synthesis ${ }^{12-15}$ and promote the differentiation of naive Th cells to Th1 lymphocytes. IL-18 (IFN- $\gamma$-inducing factor, or IGIF) is produced by monocyte- and macrophage-like cells, acts alone or synergistically with IL-12 to augment IFN- $\gamma$ release by T cells and

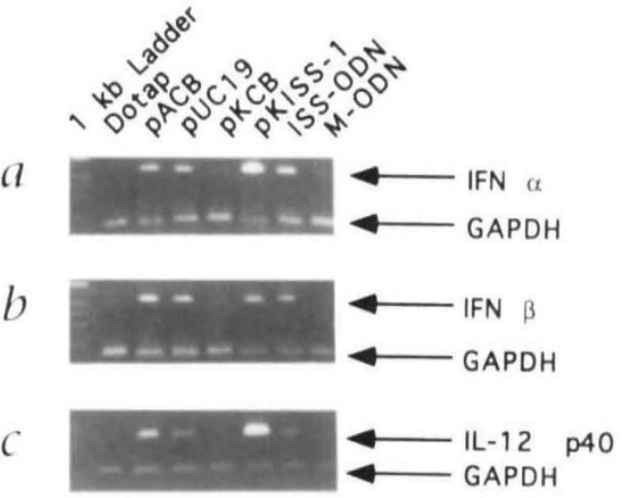

Fig. 5 Messenger RNA levels of the IFN- $\gamma$ inducers IFN$\alpha(a)$, IFN- $\beta(b)$, IL-12 p40 (c), and IL-18 (d) after transfection of fresh human macrophages by the 5'-AACGTT-3' ISS PDNAS (PACB, pUC19, and pKISS-1) and the ISS-ODN versus transfection of ISS-deficient PDNA (pKCB) or M-ODN. Induction of IL-12 p40 (e) and
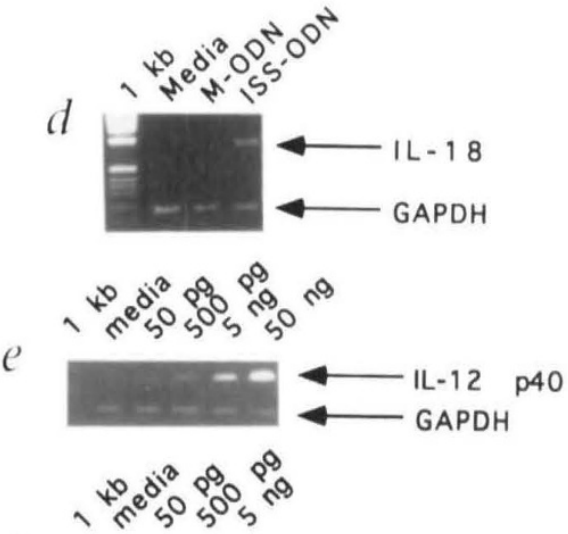

$\mathrm{IL}-18(f)$, mRNAs in human macrophages by IFN- $\alpha$. Cells

were incubated with the indicated amounts of hIFN-a (Biosource) for $3 \mathrm{~h}$. RT-PCR of the GAPDH mRNA is shown as a control for the quantity and integrity of RNA. DNA molecular size markers (1-kb DNA ladder, Gibco BRL) are indicated in kilobases. The GAPDH PCR product was electrophoresed for $5 \mathrm{~min}$ before loading. The data shown represent one of three similar experiments performed on macrophages obtained from three different healthy donors.
Table 2 Production of cytokines involved in Th1 differentiation by hPBMCs untransfected (medium only) or transfected with ISS-ODN, M-ODN or polyinosinic-polycytidilic acid (pl:C)

\begin{tabular}{|c|c|c|c|c|}
\hline \multicolumn{5}{|c|}{ Cytokines produced $(\mathrm{pg} / \mathrm{ml})$ for conditions } \\
\hline & Medium & ISS-ODN & M-ODN & pl:C \\
\hline hIFN- $\gamma$ & $7 \pm 4$ & $222 \pm 47$ & $38 \pm 15$ & $140 \pm 43$ \\
\hline hIFN- $\alpha$ & $<4$ & $442 \pm 40$ & $37 \pm 15$ & $101 \pm 32$ \\
\hline hIL-12 & $29 \pm 3$ & $82 \pm 8$ & $48 \pm 6$ & $71 \pm 11$ \\
\hline hIL-2 & $11 \pm 4$ & $30 \pm 10$ & $22 \pm 8$ & $32 \pm 4$ \\
\hline hTNF- $\alpha$ & $25 \pm 12$ & $4 \pm 2$ & $6 \pm 3$ & $4 \pm 3$ \\
\hline hIL-4 & $16 \pm 3$ & $13 \pm 2$ & $15 \pm 2$ & $21 \pm 2$ \\
\hline
\end{tabular}

Results are the means \pm s.e.m obtained from PBMCs of eight healthy volunteers. Transfection of bulk BALB/C splenocytes, under the same conditions induced $1119 \pm$ $227 \mathrm{pg} / \mathrm{ml}$ of IFN- $\gamma$ for ISS-ODN and less than $5 \mathrm{pg} / \mathrm{ml}$ for M-ODN. The murine IL-4 levels for both ISS-ODN or M-ODN were below background $(<4 \mathrm{pg} / \mathrm{ml})$.

enhances NK-cell cytolytic activity ${ }^{15}$. Thus, the ISSs activate the precise cytokine network required to induce an initial burst of IFN- $\gamma$ in an antigen-independent fashion (Table 2 and Fig. 6). In the presence of a protein antigen, this response promotes the differentiation of naive $\mathrm{CD}_{4}{ }^{+} \mathrm{T}$ cell toward Th1 cells, leading to a second burst of IFN- $\gamma$ production, this time in an antigen-dependent fashion (Table 1 and Fig. 6). Thus, this basic mechanism of ISS activation can be harnessed to evoke a Th1 response to a coadministered protein antigen.

Notably, the ISSs were initially discovered in the mycobacterial genome as DNA sequences that selectively enhanced NK cell activity ${ }^{16}$. Based on empirical results, Freund utilized mycobacterial extract as a major constituent in his adjuvant formula ${ }^{23}$. The data of this study suggest that the potent immunostimulatory effects of Freund's adjuvant may depend on the ISS-enriched mycobacterial DNA (ref. $7,16,23$ ) in addition to the cell-wall ingredients such as lipids and protein ${ }^{24}$. It is of interest that the in vivo administration of ISS-pDNA (that is, pUC19), in contrast to complete Freund's adjuvant, does not induce inflammatory reactions in the injected site ${ }^{25}$. Thus, the ISS-pDNA adjuvant can provide, in part, the immunostimulatory effects of the Freund's adjuvant without the severe inflammatory and toxic side effects attributed to the paraffin oil and the mycobacterial cell-wall products ${ }^{24}$.

These data support the idea that higher organisms can combat an invading pathogen by recognition of particular polynucleotide sequences. These include foreign RNA (for example, double-stranded RNA) or DNA (that is, ISS). In contrast to bacteria, which utilize restriction enzymes to eliminate an invading pathogen (for example, phage), the recognition of these nucleic acid sequences activates innate immunity $^{26-29}$ and, subsequently, the adaptive immune response as well, in order to eliminate the "dangerous DNA" and its products. In this respect, the ISSs represent an immune "danger/alarm-like signal" ${ }^{30}$ and trigger the release from transfected APCs and NK cells of a consorted set 
a Innate Immune Response (Antigen Independent)

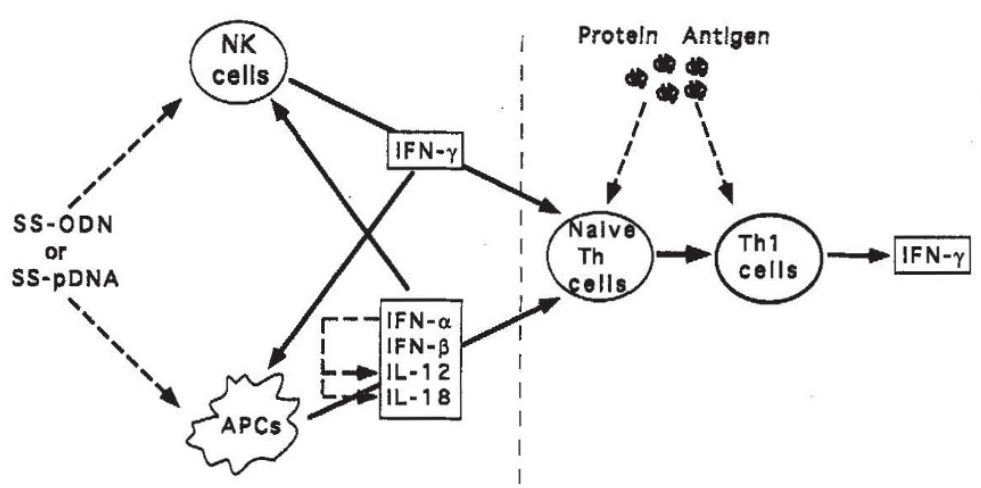

Initial Burst of IFN- $\gamma$ b Adaptive Immune Response (Antigen Dependent)

Second Burst of IFN- $\gamma$
Fig. 6 The postulated instructive role of innate immunity activated by the ISSs on the subsequent Th1 differentiation $^{31}$ in this system (ISS-DNA/antigen coadministration). $a$, ISS-ODN or ISS-pDNAs trigger the release of IFN- $\gamma$ and IFN- $\gamma$-inducers ("dangerkines") by natural killer cells (NK) and antigen-presenting cells (APCs) (for example, macrophages), respectively. These responses result in the initial burst of IFN- $\gamma$ in the picogram/milliliter range (see Table 2) in an antigen-independent fashion. IFN- $\alpha$ has an amplification role in IL-12 and IL-18 synthesis (Fig. 4). The release of IL-12 from APCs results in further IFN- $\gamma$ production by $T$ cells $^{33}$. $b$, in the presence of an antigen, the secreted innate cytokines prime naive Th cells to differentiate toward Th1 cells that produce additional IFN- $\gamma$ in the nanogram/milliliter range (see Table 1 ) in an antigen-dependent fashion. Vectors for gene vaccination provide both the adjuvant (ISS) and the genetic information for the encoded antigen'. of cytokines (which may be designated "dangerkines") that inhibit pathogen replication, activate cell-mediated immunity and instruct the adaptive immune system to differentiate toward a protective Th1 response ${ }^{31}$. The DNA adjuvants achieve this effect by mimicking an intracellular pathogen without the risks posed by real infection.

In summary, the coadministration of ISS-DNAs with a conventional vaccine not only markedly augments antibody production, but also activates cell-mediated immunity. Protein/ISS-DNA combinations might thus constitute optimal vaccines with which to combat infectious pathogens and to desensitize against allergens.

\section{Methods}

Polynucleotide-based reagents. The PACB plasmid vector is a pUC19based plasmid (ampR) containing the cytomegalovirus (CMV) promoter/enhancer sequences (807-bp), along with the CMV immediate early intron (824-bp) and a 560-bp bovine growth hormone poly(A) signal $(B G H p A)$. The $p K C B$ vector was constructed by replacing the 1542 -bp $\beta$ lactamase-containing fragment (ampicillin resistance) (BspHI-HindIII) of pACB with a 1042-bp kanamycin resistance-containing fragment (BspHI-HindIII). The pKISS-0 vector was constructed by EcoRI restriction digestion, followed by religation of the backbone at the EcoRI sites, thus removing the expression vector cassette [from the CMV promoter to the poly(A)+]. The pKISS-1 vector was constructed by Psp1406I restriction digestion, followed by religation of the Psp1406/ backbone, resulting in the removal of the expression vector cassette. The AACCTT sequences in the resultant vectors were confirmed by Psp1406I digestion. The pKISS- 2 vector was made by ligation of double-stranded ISS-oligo (5'-3': sense, CATGAACGTTCCC; antisense, CATCGCGAACGTT) into the BspHI site of pKISS1 , disrupting the $B s p H I$ site at one end. The pKISS-3 vector was made by ligation of double-stranded ISS-ODN (5'-3': sense, GTGCGAACCTTCAACGTTCCC; antisense, CCACCCCAACCTTCAACCTTC) into the Banl site of pKISS-2, disrupting both Banl sites, and adding a HindIII site. The PACB-LacZ vector (encoding $\beta$-gal) has been described elsewhere ${ }^{4}$.

Escherichia coli and calf thymus genomic DNA as well as polyinosinicpolycytidilic acid ( $\mathrm{pl:C}$ ) were purchased from Sigma Chemical Co. The genomic DNAs were sonicated and purified from endotoxin as described below.

For transfection of hPBMCs we used phosphodiester double-stranded ODNs. The sequences are ISS-ODN, 5'-TCATTGCAAAACCTTCTTCGCGCCC-3' (adapted from pUC19 sequence 2288-2312, containing the putative immunostimulatory 5'-AACCTT-3' palindrome); the mutated (M)-ODN, 5'-TCATTCGAAAACCTTCTTCCGCGCG-3', were purchased from Integrated DNA Technologies Inc. (Coralville, IA).

For protein/DNA coimmunization we used phosphodiester and phosphorothioate, single-stranded ODNs. The sequences are ISS-ODN,
5'-TGACTGTGAACGTTCGAGATGA-3', M-ODN, 5'-TGACTGTGAAGGTTACACATGA-3' were purchased from Trilink (San Diego, CA).

Plasmid DNAs were prepared using Qiagen Maxi Kits (Chatsworth, CA). Endotoxin was removed by Triton X-114 (Sigma) extractions as described previously ${ }^{3,4}$. Endotoxin levels in all the nucleic acid preparations were determined using the Pyrotell limulus amebocyte lysate (LAL) assay (Assoc. Cape Cod, Woods Hole, MA $)^{3,4}$ and were below $2 \mathrm{ng} / \mathrm{mg}$ DNA. Before injection, all the polynucleotide-based reagents were precipitated in $100 \%$ ethanol, washed in $70 \%$ ethanol and dissolved in normal saline.

Transfection of hPBMCs and mouse splenocytes. Heparinized peripheral blood from eight healthy donors was diluted 1:2 with RPMI 1640. PBMCs were isolated by Ficoll Hypaque (Sigma) gradient, washed twice with RPMI 1640 , resuspended at $2 \times 10^{6} \mathrm{ml}$ in RPMI $1640 / 1 \%$ FBS (low endotoxin), in Teflon tubes and incubated for $14 \mathrm{~h}$ at $37{ }^{\circ} \mathrm{C}$, in a $5 \% \mathrm{CO}_{2}$ incubator. Transfections of ISS-ODN, M-ODN and pl:C (10 $\mu \mathrm{g}$ each) were performed with DOTAP (Boehringer Mannheim). Twenty-four hours after transfection, supernatants from transfected and untransfected wells were collected. The levels of IFN- $\gamma$, IFN- $\alpha$, IL-12, IL-2 and IL- 4 were detected by commercial ELISA kits (Biosource, Camarillo, CA). Transfection efficiencies (90\%) were confirmed by cytofluorometric analyses of human CEM lymphoblasts transfected with fluorescein-ISS-ODN. The same procedure was used for bulk BALB/C splenocytes. In another set of experiments hPBMCs from three different donors were fractionated to $C D 4^{-}$and $C D 8^{+}$subpopulations $\left(C D 4^{+}\right.$ and $C D 8^{+}$cell separation columns, R\&D Systems, Minneapolis, MN). The enriched $\mathrm{CD}^{+}$and $\mathrm{CD}^{+}$subpopulations ( $>90 \%$ purity for each subset as confirmed by cytofluorometric analyses), as well as the human $T$ cell lines CEM and Jurkat, were transfected according to the protocol mentioned above. Mouse IFN- $\gamma$ was detected in the supernatants of transfected splenocytes as was previously described ${ }^{3,4}$.

Immunizations protocols, antlbodles and cytokine assays. Female $B A L B / C$ mice (6-8 weeks of age) were injected intradermally (i.d.) at the base of the tail once, or three times, 2 weeks apart, with $10 \mu \mathrm{g}$ of $\beta$-gal (Calbiochem, San Diego, CA) or mixed with $50 \mu \mathrm{g}$ of pDNA or $50 \mu \mathrm{g}$ of ODNs in $50 \mathrm{ml}$ normal saline. Influenza hemagglutinins (HGNs, $15 \mu \mathrm{g}$ influenza virus vaccine, trivalent, types $A$ and $B$, subvirion antigen, 1995-96 formula, Wyeth-Ayerst Laboratories, Philadelphia, PA) were injected alone, or with pKISS-0, pKISS-3, ISS-ODN (phosphorothioate) or with M-ODN (phosphorothioate) $(50 \mu \mathrm{g}$ for each DNA preparation) under the same conditions. Gene vaccinated mice ( $P A C B-L a C Z)$ were immunized i.d. with (50 $\mu \mathrm{g}$ ) once or three times, at 2-week intervals. For IgE induction, mice were primed i.d. with $10 \mu \mathrm{g} \beta$-gal mixed with various DNAs $(50 \mu \mathrm{g})$. These include $\beta$-gal/pKISS-0, $\beta$-gal/pKISS-3, $\beta$-gal/M-ODN (phosphorothioate), $\beta$ gal/ISS-ODN (phosphorothioate), or with saline. Four weeks after priming they were injected intraperitoneally with $2 \mu \mathrm{g} \beta$-gal in $3 \mathrm{mg}$ alum as previously described ${ }^{3}$ and followed for another 7 weeks. Anti- $\beta$-gal antibody assays ( $\lg G, \lg G 1, \lg G 2 a$ and $\lg E$ ) were performed by ELISA or 
radioimmunoassay as was previously described ${ }^{3,4}$. Anti-influenza $\mathrm{HGN}$ antibody screens $(\operatorname{lgC}, \operatorname{lgG} 1, \lg \mathrm{G} 2 \mathrm{a}$ ) were similarly performed using the original vaccine as the coated antigen ( $5 \mu \mathrm{g}$ of $\mathrm{HGN} / 1 \mathrm{ml}$ of BBS).

Cytokine release (IFN- $\gamma$ and IL-4) by $\beta$-gal-stimulated splenic CD4 ${ }^{+} T$ cells from $\beta$-gal, $\beta$-gal/pDNAs and from PACB-LacZ (gene vaccine) immunized mice (three i.d. immunizations for each group) was performed as previously described ${ }^{4}$

Reverse transcriptase-PCR studies. Human (h) macrophages were isolated from the hPBMCs of three donors by fibronectin adherence, as previously described ${ }^{32}$. Transfections were performed as described above, except that the medium was RPMI 1640 supplemented with $5 \%$ low endotoxin FBS and that $2.5 \mu \mathrm{g}$ of pDNA(s) or double-stranded ISS-ODN or doublestranded M-ODN were added to $2310^{5}$ cells per Teflon tube (Pierce, Rockford, IL). Three hours after transfection, mRNA was extracted using RNA STAT-60 ("B"; Tel Test, Inc., Friendswood, TX) and analyzed by RTPCR. ODNs used in the RT-PCR assays were as follows: hIFN- $\alpha$ (430-bp PCR product, sense primer, 5'-TTTCTCCTCCCTCAAGCACAG-3', antisense primer, 5'-GCTCATGATTTCTCCTCTCACA-3'), hIFN- $\beta$ (380-bp PCR product, sense primer, 5'-AAAGAAGCACCAATTTTCAGC-3', antisense primer, 5'-CCTTGGCCTTCAGGTAATGCA-3'), hIL-12 p40 (181-bp PCR product, sense primer, 5'-GGTGGTCCTCACCTGTCACA-3', antisense primer, 5'-GTGAAGCAGCACGAGCGAATG-3) (positions 128 to 308 of the IL-12 p40 CDNA), hIL-18 (IGIF) primers (866-bp PCR product, sense primer, 5'-CTTCCTCTCGCAACAAACTATT, antisense primer, 5'-GCCTCACCACAACCTCTACCTC) and hGAPDH as controls (190-bp PCR product, 5' primer, 5'-TGGTATCGTGGAAGGACTCATGAC; 3' primer, 3'-ATCCCAGTGAGCTTCCCGTTCAGC).

Synthesis of single-stranded DNA from mRNA was performed with a Superscript kit (Gibco BRL, Gaithersburg, MD). PCR was performed using Taq DNA polymerase (Boehringer Mannheim), buffer $F$ of the PCR Optimization Kit (Invitrogen, San Diego, CA), $5 \mathrm{ng} / \mathrm{ml}$ of each primer per reaction, and the following PCR conditions: $94{ }^{\circ} \mathrm{C}$ for $1 \mathrm{~min}, 58{ }^{\circ} \mathrm{C}$ for 1 min, $72^{\circ} \mathrm{C}$ for $30 \mathrm{~s}, 35$ cycles and a final extension step of $72^{\circ} \mathrm{C}$ for $7 \mathrm{~min}$. The strength of the band of the GAPDH PCR product (housekeeping gene) was used as an internal standard. PCR analysis was performed by UV visualization of PCR products after size separation (electrophoresis) on a $1.5 \%$ agarose gel. Electrophoresis was performed in $0.5 \%$ TBE buffer in the presence of ethidium bromide ( $1 \mathrm{mg} / \mathrm{ml}$ agarose). Size determination was confirmed by comparison with the sizes of DNA markers using the $1-\mathrm{kb}$ ladder (Gibco BRL).

\section{Acknowledgments}

The authors thank P. Charos, S. Malek, P. Cheng, C. Gray and I. Carroll for their excellent technical assistance and N. Noon and J. Van Uden for editorial assistance. This work was supported in part by grants Al 37305, Al 29614 and AR 41897 from the National Institutes of Health, Al 27670, Al 38858, HL 57911 and Al 36214 for AIDS Research, the Research Center for AIDS and HIV infection of San Diego Veterans Affairs Medical Center as well as the UCSD Center for AIDS Research (CFAR) development grant (Al 36214) and Dynavax Technologies Corporation. M.R. was supported by an NIH Training Grant AI 07384. E.M.-O. is supported by the Spanish Ministry of Culture and Education, and J.G. is a Howard Hughes Medical Institute Medical Student Research Training Fellow.

\section{RECEIVED 29 MAY; ACCEPTED 4 JUNE 1997}

1. Manickan, E., Rouse, R.J., Yu, Z., Wire, W.S. \& Rouse, B.T. Genetic immunization against herpes simplex virus. Protection is mediated by $\mathrm{CD}^{*} \mathrm{~T}$ lymphocytes. $)$ Immunol. 155, 259-265 (1995).

2. Xiang, Z. \& Ertl, H.C. Manipulation of the immune response to a plasmid-encoded viral antigen by coinoculation with plasmids expressing cytokines. Immunity 2 , 129-135 (1995).

3. Raz, E. et al. Preferential induction of a Th1 immune response and inhibition of specific IgE antibody formation by plasmid DNA immunization. Proc. Natl. Acad. Sci.
USA 93, 5141-5145 (1996).

4. Broide, D., Martin-Orozco E., Roman, M., Carson., D.A. \& Raz E. Intradermal gene vaccination down-regulates both arms of the allergic response. I. Allergy Clin. immunol. 99, s129 (1997).

5. Sato, $Y$. et al. Immunostimulatory DNA sequences necessary for effective intradermal gene immunization. Science 273, 352-354 (1996).

6. Bird, A.P. CpG islands as gene markers in the vertebrate nucleus. Trends Genet. 3 , 342-347 (1987).

7. Yamamoto, S. et al. Unique palindromic sequences in synthetic oligonucleotides are required to induce IFN and augment IFN-mediated natural killer activity. 1. Immunol. 148, 4072-4076 (1992).

8. Mosmann, T.R. \& Coffman, R.L. TH1 and TH2 cells: Different patterns of lymphokine secretion lead to different functional properties. Annu. Rev. Immunol. 7 , 145-173 (1989).

9. Raz, E. et al. Intradermal gene immunization: The possible role of DNA uptake in the induction of cellular immunity to viruses. Proc. Natl. Acad. Sci. USA. 91, 9519-9523 (1994).

10. Condon, C., Watkins, S.C., Celluzzi, C.M., Thompson, K. \& Falo, L.D., Ir. DNAbased immunization by in vivo transfection of dendritic cells. Nature Med. 2, 1122-1128 (1996).

11. Corr, M., Lee, D.J., Carson, D.A. \& Tighe, H. Gene vaccination with naked plasmid DNA: Mechanism of CTL priming. J. Exp. Med. 184, 1555-1560 (1996).

12. Brinkmann, V., Geiger, T., Alkan, S. \& Heusser, C.H. Interferon alpha increases the frequency of interferon gamma-producing human CD4 ${ }^{+}$T cells. J. Exp. Med. 178, 1655-1663 (1993).

13. Yaegashi, Y., Nielsen, P., Sing, A., Galanos, C. \& Freudenberg, M.A. Interferon beta, a cofactor in the interferon gamma production induced by gram-negative bacteria in mice. 1. Exp. Med. 181, 953-960 (1995).

14. Trinchieri, G. Interleukin-12: A proinflammatory cytokine with immunoregulatory functions that bridge innate resistance and antigen-specific adaptive immunity. Annu. Rev. Immunol. 13, 251-276 (1995).

15. Okamura, $\mathrm{H}$. et al. Cloning of a new cytokine that induces IFN-gamma production by T cells. Nature 378, 88-91 (1995).

16. Kimura, $Y$. et al. Binding of oligoguanylate to scavenger receptors is required for oligonucleotides to augment NK cell activity and induce IFN. 1. Biochem. 116, 991-994 (1994).

17. Cowdery, I.S., Chace, I.H., Yi, A.K. \& Krieg, A.M. Bacterial DNA induces NK cells to produce IFN-gamma in vivo and increases the toxicity of lipopolysaccharides. 1 . Immunol. 156, 4570-4575 (1996).

18. Graham, B.S. \& Wright, P. Candidate AIDS vaccine. N. Engl. I. Med. 333 , 1331-1339 (1995).

19. Burke, R.L. Current status of HSV vaccine development. in The Human Herpesviruses (eds. Roizman, B., Whitley, R.J. \& Lopez, C.). p 367 (Lippincott-Raven, Philadelphia, 1993).

20. Pertmer, T.M., Roberts, T.R. \& Haynes, J.R. Influenza virus nucleoprotein-specific immunoglobulin $\mathrm{G}$ subclass and cytokine responses elicited by DNA vaccination are dependent on the route of vector DNA delivery. J. Virol. 70, 6119-6125 (1996).

21. Feltquate, D.M., Heaney, S., Webster, R.G. \& Robinson, H.L. Different Thelper cell types and antibody isotypes generated by saline and gene gun DNA immunization. f. Immunol. 158, 2278-2284 (1997).

22. Fynan, E.F. et al. DNA vaccines: protective immunizations by parenteral, mucosal, and gene-gun inoculations. Proc. Natl. Acad. Sci. USA 90, 11478-11482 (1993).

23. Freund, I., Casals, J. \& Hosmer, E.P. Sensitization and antibody formation after injection of tubercle bacilli and paraffin oil. Proc. Soc. Exp. Biol. Med. 37, 509-513 (1937).

24. Munoz, J. Effect of bacteria and bacterial products on antibody response. Adv. immunol. 4, 397-440 (1964)

25. Raz, E. et al. Cationic lipid inhibit intradermal genetic vaccination. in: Vaccine 94 71-75 (Cold Spring Harbor Laboratory Press, Cold Spring Harbor, NY, 1994).

26. Pisetsky, D.S. Immune activation by bacterial DNA; A new genetic code. Immunity 5, 303-310 (1996).

27. Krieg, A.M. et al. CpG motifs in bacterial DNA trigger direct B-cell activation. Nature 374, 546-549 (1995).

28. Haipern, M.D., Kurlander, R.J. \& Pisetsky, D.S. Bacterial DNA induces murine interferon-gamma production by stimulation of interleukin-12 and tumor necrosis factor-alpha. Cell. Immunol. 167, 72-78 (1996).

29. Klinman, D.M., Yi, A.K., Beaucage, S.L., Conover, J. \& Krieg, A.M. CpG motifs present in bacteria DNA rapidly induce lymphocytes to secrete interleukin 6 , interleukin 12, and interferon gamma. Proc. Natl. Acad. Sci. USA 93, 2879-2883 (1996).

30. Matzinger, P. Tolerance, danger, and the extended family. Annu. Rev. Immunol. 12, 991-1045 (1994).

31. Fearon, D.T. \& Locksley, R.M. The instructive role of innate immunity in the acquired immune response. Science 272, 50-53 (1996).

32. Geng, Y., Gulbins, E., Altman, A. \& Lotz, M. Monocyte deactivation by interleukin 10 via inhibition of tyrosine kinase activity and the Ras signaling pathway. Proc Nati. Acad. Sci. USA 91, 8602-8606 (1994).

33. Bradley, L.M., Dalton, D.K. \& Croft, M. A direct role for IFN-gamma in regulation of Th1 cell development. /. Immunol. 157, 1350-1358 (1996). 